\title{
Neue Leitlinie in diesem Jahr erwartet
}

— Schon heute leiden in Deutschland 6,3 Millionen Menschen an Knochenschwund, davon 5,3 Millionen Frauen über 50 Jahre. Der DVO rechnet mit einer Verdopplung bis 2025. „Die Inzidenz wird wahrscheinlich unterschätzt, da ein Großteil der Patienten unterdiagnostiziert und unbehandelt ist", mutmaßt Prof. Dr. Peyman Hadji, Marburg. Deutschland gehört unter den Industrieländern zu den Schlusslichtern: Nur ein Viertel der Betroffenen erhält eine osteoporosespezifische Behandlung.

\section{Hohe Therapietreue}

Dabei gibt es wirksame Therapien, z. B. Denosumab (Prolia ${ }^{\circledR}$ ), das in zahlreichen Leitlinien mit AAA-Evidenzgrad klassifiziert wurde und durch geringe Nebenwirkungen auffällt. Der RANK-Ligand-Inhibitor
Denosumab wirkt sowohl am vertebralen als auch am nicht-vertebralen Knochen insbesondere an der Hüfte. Die verbesserte Knochendichte sei durch eine Phase-IIIStudie (Freedom-Studie) bei 7800 postmenopausalen Frauen mit Osteoporose belegt.

Handlungsbedarf sieht Hadji insbesondere in der Therapietreue. Unter den oralen Bisphosphonaten sei zu beobachten, dass etwa zwei Drittel der Patienten spätestens nach einem Jahr die Einnahme abbrechen. Bei Denosumab liege die Therapietreue über zwölf Monate bei $75 \%$.

Auf die besseren trabekulären und kortikalen Effekte - etwa im Vergleich zu Alendronat - wies Prof. Dr. Matthias Schiecker, München, hin. Auch langfristig bleibe das Frakturrisiko an der Hüfte über mindestens sechs Jahre auf einem niedrigen Niveau. Aktuelle Vergleichsstudien mehrerer Wirkstoffe belegen laut Prof. Dr. Christopher Niedhart, Heinsberg, eine bessere Eignung bei multimorbiden Patienten. Insbesondere bei Niereninsuffienz sei Denosumab besser geeignet, da es nicht weiter verstoffwechselt werde.

Der DVO hat in Weimar eine Neufassung der Leitlinie von 2009 beschlossen. Die finale Fassung wird im Herbst 2013 erwartet. Die wohl wichtigste Neuerung ist die Abkehr vom 10-Jahres-Frakturrisiko zu einem Einjahreszeitraum. Wie das allerdings in die klinische Praxis umgesetzt werden kann, muss noch geklärt werden.

- Robert Büssow

Quelle: OsteologieTagung der DGO 2013, Weimar, März 2013 (Veranstalter: Amgen und GSK)

\section{Allergische Sensibilisierung und Entzündungsmarker im Visier}

Allergische Sensibilisierung, Wheezing und Asthma sind eng miteinander verknüpft. Über die zeitliche Abfolge und die immunologischen Zusammenhänge weiß man bisher allerdings noch nicht sehr viel. Der Preisträger des mit 30000 Euro dotierten „International Klosterfrau Award for Research of Airway Diseases in Childhood 2013", Dr. Daniel James Jackson, Pädiater an der UniversitätsklinikWisconsin/Oregon (USA), hat durch seine klinischen und experimentellen Studien einige offene Fragen auf diesem Gebiet beantwortet und die klinische Praxis bzw. die wissenschaftliche Forschung vorangebracht.

Jackson untersuchte den zeitlichen Zusammenhang zwischen allergischer Sensibilisierung und Wheezing bei viralen Atemwegsinfekten bei Kindern bis zu einem Alter von sechs Jahren. In seiner prospektiven Longitudinalstudie mit Neugeborenen von Müttern mit Asthma gewann er folgende Erkenntnisse: Die allergische Sensibilisierung gegen Aeroallergene ist bei den Kleinkindern die erste nachweisbare pathologische Veränderung. Erst bei Vorliegen einer Aeroallergen-Sensibilisierung reagieren die Kinder bei einer Virusinfektion der Atemwege mit Wheezing, was auf pathologische Veränderungen der Bronchien hinweist. Weiterhin fand Jackson heraus, dass bei sensibilisierten Kindern nur das Humane Rhinovirus (HRV) Wheezing auslösen kann, nicht dagegen andere atemwegspathogenen Viren wie das Synzytialvirus [Jackson DJ et al. Am J Respir Crit Care Med 2012;185:281-285].

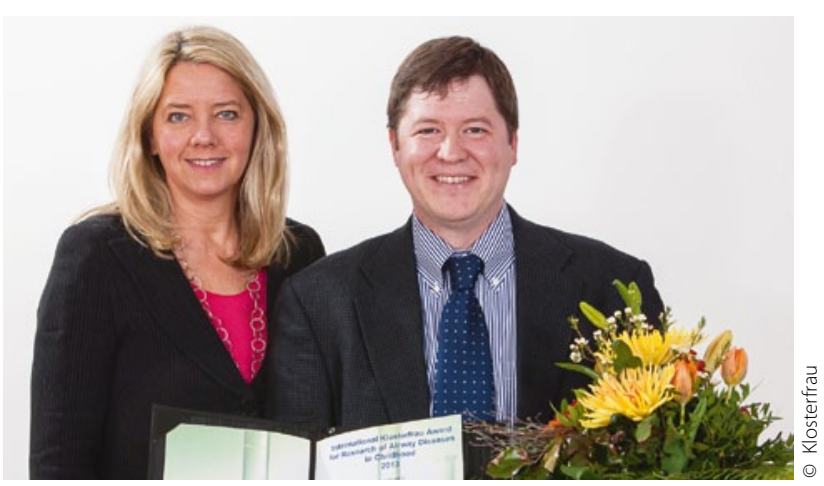

Der diesjährige Preisträger Dr. Daniel James Jackson mit der Vorsitzenden der Expertenjury, Prof. Dr. Gesine Hansen.

\section{Sensibilisierte Kinder zeigen eine verminderte} Immunabwehrreaktion

Jackson erforschte weiterhin, warum Kinder mit einer allergischen Sensibilisierung schwerere Symptome bei viralen Atemwegsinfektionen aufweisen als Kinder ohne Sensibilisierung. Seine Befunde: Die Immunzellen von sensibilisierten Kindern sind inaktiver, wenn sie in Kontakt mit Viren kommen. Die Abwehr ist nur schwach. Die Viren können die Atemwege daher stärker schädigen. Möglicherweise findet dieser Prozess auch direkt in Immunzellen des Bronchialgewebes statt [Durrani SR et al. J Allergy Clin Immunol 2012;130:489-495].

- Nach Informationen des Unternehmens Klosterfrau Quelle: 35. Tagung der Gesellschaft für Pädiatrische Pneumologie, März 2013, Lübeck 